\title{
Anesthetic neurotoxicity: an emerging role for glia in neuroprotection
}

\author{
Josh D. Bell ${ }^{1}$ - Creed M. Stary ${ }^{2}$
}

Published online: 1 March 2017

(C) Springer-Verlag Berlin Heidelberg 2017

Neurotoxicity associated with anesthetics and surgery remains a relevant issue in clinical perioperative care. In the past 15 years, preclinical data in rodents has widely shown that volatile anesthetics induce neuronal apoptosis and impair synaptogenesis in the developing brain, raising suspicion that childhood exposure to anesthetics may confer some degree of permanent cognitive impairment [1]. Confounding variables, including patient comorbidities and the physiologic response to the surgery itself, has complicated a precise determination of the clinical influence of anesthetics on neurodevelopment in children and adolescents, and the topic remains widely debated. Anesthetics have also been implicated in adult postoperative cognitive decline (POCD). The morbidity associated with POCD is large, accounting for increased time to discharge, increased mortality in the first year after surgery, rising public health costs, and susceptibility to delirium and its associated sequelae [2]. POCD is associated with poor compliance with rehabilitation, altered levels of consciousness, and complicates the postoperative administration of opioid analgesics (particularly the use of patient-controlled analgesia). Systematic reviews suggest POCD occurs in $>10 \%$ of the noncardiac surgery population over 60 years of age [3]. Undoubtedly, how anesthesia and surgery may lead to cognitive impairment in some patients, both young and old, is a complex issue.

Creed M. Stary

cstary@stanford.edu

1 Department of Anesthesia, University of Toronto, Toronto, ON, Canada

2 Department of Anesthesiology, Perioperative and Pain Medicine, Stanford University School of Medicine, 300 Pasteur Drive, MC5117, Stanford, CA 94305, USA
In this issue of the Journal of Molecular Medicine, Gui and coauthors shed interesting new light on the role of glial cells in anesthetic neurotoxicity. Glia, including microglia and astrocytes, are nonneuronal cells increasingly recognized for the major roles they play in neuronal development and maintenance in health and disease. Microglia share a common myeloid lineage with monocytes and macrophages, and similarly function in pathogen recognition, phagocytosis (of damaged cells, inactive synapses, debris, and infectious agents), and induction of inflammatory mediators. Astrocytes regulate cerebral circulation, extracellular ionic homeostasis, and release of energy substrates in the brain. In addition to their role in neuronal housekeeping and protection, astrocytes regulate neurotransmission and synapse formation. The neuronal milieu within the brain is supported by the interaction of trophic factors secreted by neurons and glia that mediate synaptic maturation, axonal sprouting during development, and repair following injury. We have previously demonstrated that astrocytes can protect developing neurons from volatile anesthetic neurotoxicity by regulating brain-derived neurotrophic factor, a neurotrophin secreted by neurons [4]. In the present study, Gui et al. further advance the prospect of a central role for astrocytes in protection from anesthetic neurotoxicity by focusing on glial-derived neurotrophic factor (GDNF), a neurotrophin secreted by astrocytes, which plays a critical role in neuronal development and in neuronal protection following injury (Fig. 1). GDNF is currently being explored as a therapeutic target in a number of nervous system disorders, including Parkinson's disease; however, the role of GDNF in neonatal anesthetic neurotoxicity and the therapeutic potential of GDNF for prevention of adult POCD has not been previously explored.

In their investigations, Gui et al. employed a combined anesthesia-surgery model in neonatal rats, utilizing the most commonly used anesthetic in clinical practice (sevofluorane), and 


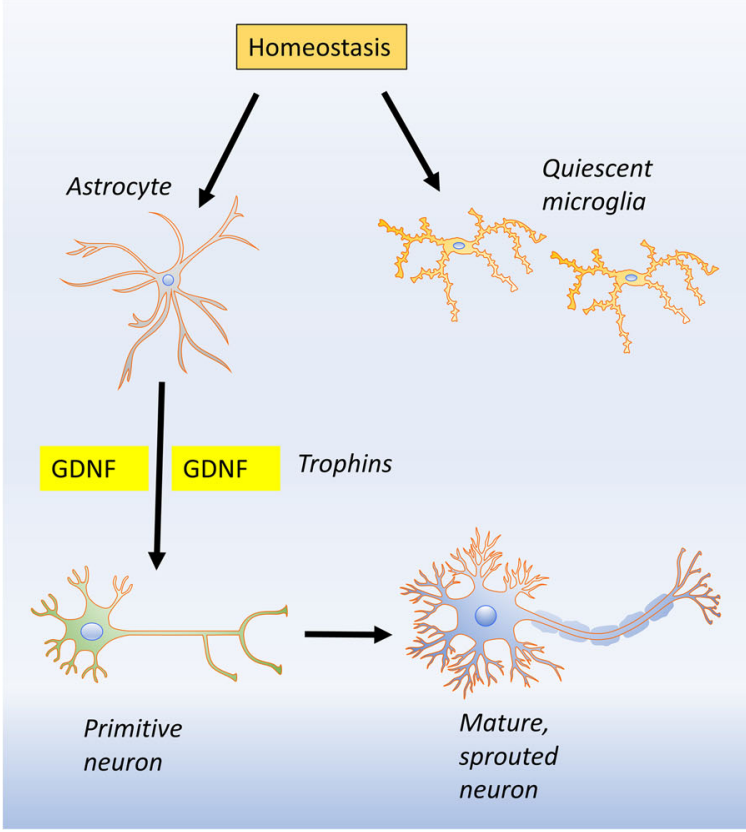

Fig. 1 The role of GDNF in anesthesia/surgery-related neurodegeneration. (Left panel) In homeostasis, GDNF, secreted by astrocytes, promotes neuronal maturation, synaptic sprouting, and repair from injurious stimuli. Microglia, while present, are inactive and nonsecretory. (Right panel) Anesthesia and surgery in combination promotes the activation of microglia, which inhibits GDNF release from astrocytes via activation of NF-KB. GDNF antagonism reduces neuronal repair, leading to

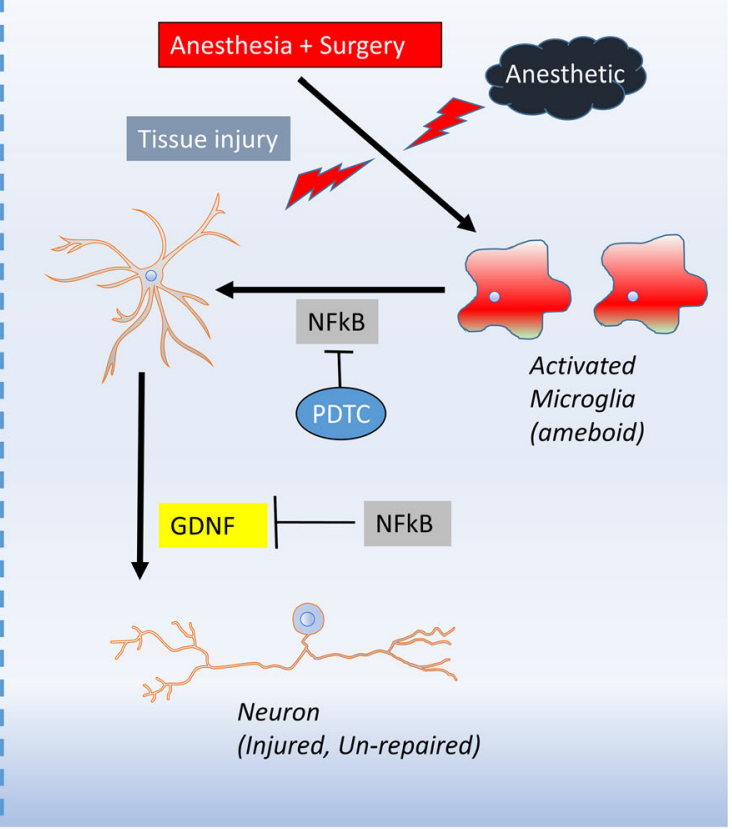

postoperative cognitive dysfunction and anesthesia/surgery-related neurotoxicity. Anti-inflammatories that antagonize NF-kB, such as PDTC, restore GDNF secretion and improve cognitive outcome. Restoration of cognitive function following anesthesia/surgery can also be achieved by exogenous application of GDNF. GDNF glial-derived neurotrophic factor, $N F-\kappa B$ nuclear factor-kappa B, PDTC pyrrolidine dithiocarbamate

prevent glial cells from appropriately responding to an inflammatory milieu. Interestingly, in the present study, inactivation of GDNF alone (without surgery) also resulted in learning and memory impairment. Further verifying astrocytes as central for neuroprotection following surgical stress, and as a potential therapeutic target for POCD, the authors demonstrated: (1) inhibition of NF- $\mathrm{KB}$ activity with pyrrolidine dithiocarbamate (PDTC) augmented GDNF levels and neurogenesis and (2) either PDTC treatment or intrathecal injection of GDNF reversed the behavioral deficits associated with anesthesia and surgery.

A number of clinical investigations have pointed to attenuation of neuroinflammation as a potential therapeutic approach for POCD. For example, ketamine, a phencyclidine derivative that not only antagonizes glutamate receptors but also has a profound anti-inflammatory effect, attenuates cognitive dysfunction following cardiac surgery [6,7]. Likewise, parecoxib, a cyclooxygenase-2 inhibitor with marked anti-inflammatory effects, also prevented cognitive decline following orthopedic surgery in an elderly cohort [8]. Recently, it was further described that dexamethasone, well known for reducing neuroinflammation in traumatic brain injury and those with cerebral edema, also improved cognitive outcome following surgery [9]. However, all of these therapies are associated with undesirable side effects, ranging from psychotropic effects, impacts on wound healing and bowel anastomoses, and increased risk of adverse cardiovascular events. Exogenous trophic factor administration might be a 
gentler therapy for POCD. Indeed GDNF has been demonstrated to have a relatively good safety profile in phase 2 trials for Parkinson's disease [10]. As astrocytes in the human brain are larger, more complex, and greater in number relative to neurons than in the rodent brain, trophic therapies targeting astrocytes may provide a key factor in overcoming translational barriers between rodents and humans. While further preclinical experiments in adult and aged animal models are necessary to determine the clinical potential for GDNF as an effective therapy for POCD, the result from the present study by Gui et al. provides intriguing new data and insight into an increasingly recognized public health problem.

\section{References}

1. Sun L (2010) Early childhood general anaesthesia exposure and neurocognitive development. Br J Anaesth 105(Suppl 1):i61-i68

2. Steinmetz J, Christensen KB, Lund T, Lohse N, Rasmussen LS, Group I (2009) Long-term consequences of postoperative cognitive dysfunction. Anesthesiology 110:548-555

3. Coburn M, Fahlenkamp A, Zoremba N, Schaelte G (2010) Postoperative cognitive dysfunction: incidence and prophylaxis. Anaesthesist 59:177-184 quiz 185
4. Stary CM, Sun X, Giffard RG (2015) Astrocytes protect against isoflurane neurotoxicity by buffering pro-brain-derived neurotrophic factor. Anesthesiology 123:810-819

5. Sun Y, Huang X, Liu M, Cao J, Chen J, Wang H, Niu H, Yu Z, Yu J, Wang $T$ et al (2012) A new alternative NF-kappaB pathway mediated the neuroprotection of GDNF on 6-OHDA-induced DA neurons neurotoxicity. Brain Res 1437:38-49

6. Hudetz JA, Iqbal Z, Gandhi SD, Patterson KM, Byrne AJ, Hudetz AG, Pagel PS, Warltier DC (2009) Ketamine attenuates postoperative cognitive dysfunction after cardiac surgery. Acta Anaesthesiol Scand 53:864-872

7. Hudetz JA, Patterson KM, Iqbal Z, Gandhi SD, Byrne AJ, Hudetz AG, Warltier DC, Pagel PS (2009) Ketamine attenuates delirium after cardiac surgery with cardiopulmonary bypass. J Cardiothorac Vasc Anesth 23:651-657

8. Zhu YZ, Yao R, Zhang Z, Xu H, Wang LW (2016) Parecoxib prevents early postoperative cognitive dysfunction in elderly patients undergoing total knee arthroplasty: a double-blind, randomized clinical consort study. Medicine (Baltimore) 95:e4082

9. Valentin LS, Pereira VF, Pietrobon RS, Schmidt AP, Oses JP, Portela LV, Souza DO, Vissoci JR, Luz VF, Trintoni LM et al (2016) Effects of single low dose of dexamethasone before noncardiac and nonneurologic surgery and general anesthesia on postoperative cognitive dysfunction - a phase III double blind, randomized clinical trial. PLoS One 11:e0152308

10. Slevin JT, Gerhardt GA, Smith CD, Gash DM, Kryscio R, Young B (2005) Improvement of bilateral motor functions in patients with Parkinson disease through the unilateral intraputaminal infusion of glial cell line-derived neurotrophic factor. J Neurosurg 102:216-222 